\title{
Cromosomopatias y lenguaje: alteraciones fonético-fonológicas asociadas a una microduplicación en el cromosoma 1q21.1. Estudio de un caso
}

\author{
JONATAN M. RODRÍGUEZ \\ Palomero \\ Universidad de Cádiz \\ Facultad de Filosofia y Letras \\ Avda. Gómez Ulla, s/n \\ 11003 Cádiz \\ E-mail: jonathan.rodriguez- \\ palomero@uca.es

\begin{abstract}
CROMOSOMOPATÍAS Y LENGUAJE: ALTERACIONES FONÉTICO-FONOLÓGICAS ASOCIADAS A UNA MICRODUPLICACIÓN EN EL CROMOSOMA 1Q21.1. ESTUDIO DE UN CASO
\end{abstract}

RESUMEN: El presente trabajo aborda el estudio lingüistico de un tipo de cromosomopatia, concretamente, la microdu plicación en el cromosoma 1.21q1 (estudio de un caso). Este tipo de alteraciones genéticas es de baja prevalencia, por lo que se desconoce mucho acerca de sus características, y más aún, sobre su in fluencia en el lenguaje, habilidades comunicativas y aprendizaje. Este estudio se centra en el componente fonético-fonológico. Para llevar a cabo el estudio de este nivel, hemos utilizado una serie de tests y pruebas estandarizadas (Clinica Evaluation of Language Fundamentals (CELF); Test de Evaluación Fonológica de Laura Bosch; Registro Fonológico Inducido; Prueba de diptongos y triptongos) y muestras de habla espontánea recogidas; a través del análisis de los datos obtenidos en las pruebas, estudiaremos las al teraciones fonético-fonológicas en las producciones orales, determinando la presencia de problemas articulatorios. Finalmente, analizaremos la estructura y características de dichas producciones alteradass, destacando su influencia en la capacidad comunicativa.

PALABRAS CLAVES: Microduplicación; cromosoma 1.21q1; alteración genética; simplificación de diptongos; estructura silábica; nivel fonético-fonológico.

SUMARIO: 1 . Introducción y estado de la cuestión. 2. Antecedentes y estudio previo. 3. Clinical Evaluation of Language Fundamentals. 4. Registro Fonológico In ducido. 5. Evaluación fonético-fonológica del habla infantil. Laura Bosch. 6 Prueba fonológica de diptongos y triptongos. 6.1. Prueba fonológica diptongos. 6.2. Prueba fonológica triptongos. 7.Con clusiones y futuras lineas de investigación.

\author{
Ma SALUd Jiménez-ROMERo \\ Universidad de Córdoba \\ Facultad de Ciencias de la \\ Educación \\ C/ San Alberto Magno s/n \\ 14004 Córdoba (España) \\ E-mail: m12jirom@uco.es
}

\begin{abstract}
CHROMOSOMOPATHIES AND LANGUAGE: PHONETIC-PHONOLOGICAL ALTERATIONS ASSOCIATED WITH A MICRODUPLICATION IN THE CHROMOSOME 1Q21.1. STUDY OF A CASE
\end{abstract}

ABSTRACT: This paper deals with the linguistic study of a type of Chromosomopathy, specifically, microduplication in chromosome $1.21 \mathrm{q} 1$ (study of a case) This type of genetic alterations is of low prevalence, so much is unknown about their characteristics, and even more, about their influence on language, communication skills and learning. This study draws attention to the phoneticphonological component. In order to study this level, we have made use of a series of examinations and standardized tests, for instance: Clinical Evaluation of Language Fundamentals (CELF), Phonological Assessment Test of Laura Bosch, Induced Phonological Record, Diphthong and Triphthong Test) and spontaneous speech samples. Throughout the analysis of the data obtained in the tests, we will study the phonological alterations in oral productions, determining the presence or absence of articulatory problems. Finally, we will analyse the structures and characteristics of these altered productions, highlighting their influence on the linguistic-communicative capacity of the studied patient.

KEY WORDS: Microduplication; chromosome 1.21q1; genetic disorder; simplification of diphthongs; syllabic structure; phonological-phonological level.

SUMMARY: 1. Introduction status of work. 2. Background and previous study. 3. Clinical evaluation of the basics of language. 4. Induced Phonological Record. 5. Phonetic and Phonological assessment of children's speech. Laura Bosch. 6 . Phonological test of diphthongs and triphthongs. 6.1. Phonological test diphthongs. 6.2. Phonological test triphthongs. 7.Conclusions and future lines of research.

\author{
ANTONio BeníteZ-BURRACo \\ Universidad de Sevilla \\ Facultad de Filologia \\ C/ Palos de la Frontera s/n \\ 41004 Sevilla (España) \\ E-mail: abenitez8@us.es
}

CHROMOSOMOPATHIES ET

LANGUAGE: ALTERATIONS PHONETOPHONOLOGIQUES ASSOCIEES A UNE MICRODUPLICATION DANS LE CHROMOSOME 1Q21.1. ÉTUDE DE CAS

RÉSUMÉ: Le travail se base sur l'étude linguistique d'un type de chromosomopathie, en particulier la microduplication dans le chromosome 1.21q1 (étude de cas). Ce type de modification génétique ayant une faible prévalence, on ignore ses caractéristiques, et par conséquent son influence sur le langage, les compétences de communication et l'apprentissage. Cette étude porte sur la composante phonétique-phonologique. Pour étudier ce niveau, nous avons utilisé une série de tests et de tests standardisés (CELF), le test d'évaluation phonologique de Laura Bosch, le registre phonologique induit, des tests de diphtongues et de triphtongues, et des échantillons de mot spontanés. A travers l'analyse des données obtenues lors des tests, nous étudierons les altérations phonologiques dans les productions orales, en déterminant la présence ou l'absence de problèmes articulatoires. Enfin, nous analysons les caractéristiques des productions altérées, en les comparant avec celles d'un individu du même stade, en soulignant son influence sur la capacité de communication linguistique.

MOTS CLÉS: Microduplication ; chromosome $1.21 \mathrm{q} 1$; altération génétique ; simplification des diphtongues ; structure syllabique; niveau phonologique-phonologique.

SOMMAIRE: 1 . Introduction et état de la question. 2. Contexte et étude précédente. 3. Evaluation clinique des bases du langage. 4. Registre Phonologique Induit. 5. Évaluation Phonologique de la parole des enfants. Laura Bosch. 6. Test Phonologique des Diphtongues et des Triphtongues. 6.1. Examen Phonologique des Triphtongues. 6.2. Examen Phonologique des Diphtongues. 7. Conclusions et futurs axes de recherche. $\begin{array}{ll}\text { Fecha de Recepción } & 05 / 02 / 2019 \\ \text { Fecha de Revisión } & 19 / 03 / 2019 \\ \text { Fecha de Aceptación } & 15 / 05 / 2019 \\ \text { Fecha de Publicación } & 01 / 12 / 2020\end{array}$ 


\title{
Cromosomopatias y lenguaje: alteraciones fonético-fonológicas asociadas a una microduplicación en el cromosoma 1q21.1. Estudio de un caso
}

\author{
Jonatan M. Rodríguez Palomero, Ma Salud Jiménez-Romero \& Antonio Benítez-BurRaco
}

\section{INTRODUCCIÓN Y ESTADO DE LA CUESTIÓN}

El nacimiento de la Psicolingüística y la Neurolingüística a lo largo del siglo XX consolidó el estudio de los trastornos del lenguaje, centrándose en su mayoria en la afasiología. Sin embargo, este modelo tradicional ha sido cuestionado a la luz de los resultados obtenidos por análisis neuro-anatómicos y conductuales. Como consecuencia, los centros lingüísticos mencionados (áreas cerebrales) se han ido ampliando para completar el reclutamiento necesario de las áreas cerebrales, que la actividad lingüística hace de circuitos neuronales adicionales a los del córtex perisilviano del hemisferio izquierdo y en el córtex prefrontal, porciones lateral y ventral, contando, además, con otras regiones cerebrales que hasta la fecha estaban exentas de cualquier tarea lingüistica (Pulvermüller, 2002).

No obstante, como sostiene Benitez-Burraco, no ha podido establecerse, por el momento, ninguna correlación entre la organización funcional del cerebro y la especialización histológica, esto es, la correspondencia entre componentes del lenguaje y determinadas estructuras cerebrales, corticales o subcorticales. Por otro lado, en lo que atañe a los mecanismos moleculares responsables del desarrollo estructural y funcionamiento de los centros nerviosos, sí parece existir un programa genético que determina la forma en la que la organización cerebral está dispuesta, y, además, unos programas responsables de la aparición y el funcionamiento de determinadas regiones cerebrales (Benitez Burraco, 2007:113-114).

Finalmente, cabe destacar las diferentes aportaciones de diversos investigadores en lo que se refiere a los genes relacionados con el desarrollo y funcionamiento del lenguaje, entre los que destacamos el gen FOXP2 o "gen del lenguaje", cuya versión mutada causa trastornos motores, como la dispraxia orofacial, y lingüísticos, como problemas en el deletreo o la repetición de palabras; y otros genes "candidatos" que también participan en la producción y compresión lingüística (Benítez Burraco, 2005:10-11).

A lo largo del presente trabajo vamos a presentar y analizar los datos del habla, obtenidos a través de diferentes pruebas y tests fonético-fonológicos; de un paciente con microduplicación en el cromosoma 1q21.1. Este estudio tiene como principal objetivo la descripción y análisis de muestras de habla en las pruebas presentadas a nuestro sujeto de investigación; todo ello, arrojará datos relevantes para poder verificar la existencia o ausencia de alteraciones lingüisticas en el nivel fonético-fonológico, como consecuencia de la alteración genética que sufre. 


\section{ANTECEDENTES Y ESTUDIO PREVIO}

Nuestro estudio de caso se centra en un sujeto de 6.11 años que sufre una microduplicacion en el cromosoma 1q21.1. Esta alteración genética es el resultado de la ruptura entre dos secciones situadas en el cromosoma 1 (banda q21.1), con la posterior duplicación del material genético entre las secciones anteriores (Unique, 2013: 2). De esta forma, el sujeto pasa a tener un segmento extra de material genético, con una consecuente alteración a nivel genético. Esta afectación, que ha sido poco estudiada debido a su baja prevalencia, destaca por tener asociados ciertos síntomas como macrocefalia, rasgos autistas, alteraciones lingüístico-cognitivas, problemas de aprendizaje y comportamentales. La microduplicación detectada en nuestro sujeto ha sido heredada por la progenitora, la cual es portadora de dicha alteración genética, aunque no ha desarrollado los síntomas asociados a esta patología. En el caso de nuestro sujeto, sí se han diagnosticado los síntomas antes mencionados.

En el estudio previo realizado a nuestro sujeto, habiendo analizado la porción de cromosoma duplicada, se observó una serie de genes que se encuentran alterados; entre ellos, cabe destacar el gen ROBO1, posible candidato para la dislexia, el cual se encuentra sobreexpresado (Imagen 1). Durante dicho estudio, también se observó problemas en el nivel fonético-fonológico que han motivado la realización del presente trabajo, con el objetivo de analizar posibles alteraciones y si estas derivan de una mala articulación o no. (Benitez-Burraco et al., 2018: 6:163).

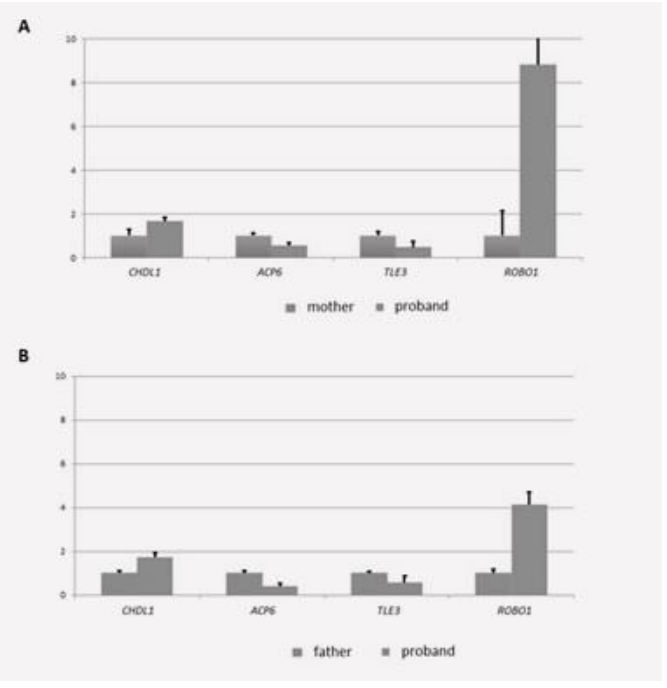

A continuación, presentamos las diferentes pruebas realizadas, acompañadas de los resultados obtenidos y el estudio de estos. 


\section{Clinical Evaluation of Language fundamentals}

La primera prueba lingüistica suministrada es la denominada Clinical Evaluation of Language Fundamentals (CELF-4) (Wiig et al., 2006). Dicha prueba, a pesar de no evaluar de forma exclusiva alteraciones fonológicas, nos puede arrojar información relevante sobre los déficits lingüístico-cognitivo presentes en nuestro sujeto, que no hayan sido detectados en el estudio previo.

Esta herramienta de evaluación lingüistica cuenta con una serie de test y sub-test que van desde la asociación de palabras (nivel léxico-semántico), hasta la evaluación de elementos pragmáticos y conversacionales (nivel pragmático-textual); gracias a los cuales, podemos estudiar las habilidades y dificultades que presenta nuestro sujeto en las situaciones comunicativas e input ofrecidos.

Las diferentes pruebas de este test están distribuidas en torno a 4 niveles de análisis:

- Nivel 1: Lenguaje básico (Language core): este nivel mide la capacidad lingüístico-comunicativa en términos generales, teniendo en cuenta las actuaciones y enunciaciones emitidas en una serie de sub-test, concretamente, Conceptos y seguir direcciones, Recordar oraciones, Construir oraciones, Clases de palabras; una vez realizadas estas subpruebas, se obtendrá una puntuación (Language Basic score) que nos ayudará a determinar la presencia o ausencia de alteraciones en el lenguaje.

Nuestro sujeto obtuvo una puntuación de 73 . Teniendo en cuenta los baremos proporcionados por la prueba, esta puntuación se traduce en una alteración del lenguaje de grado leve/moderado. Entre los sub-test con puntuaciones más bajas se encuentran: Conceptos y siguiendo direcciones, Estructuras de palabras, Formulación de oraciones, etc. Sin embargo, esta persona tiene una puntuación alta en pruebas como: Recordando oraciones, Clases de palabras receptivo y Vocabulario expresivo, situándose la puntuación del primer y el último sub-test por encima de la media, teniendo en cuenta su edad.

- El Nivel 2 de esta prueba tiene como objetivo determinar la naturaleza de la alteración o alteraciones observadas anteriormente. Dicho procedimiento se lleva a cabo a través del índice de puntuación que el sujeto obtenga en 5 sub-test:

- Lenguaje receptivo: donde se mide la capacidad del sujeto para escuchar, comprender y responder a través de acciones a inputs orales.

- Lenguaje expresivo: donde se evalúa la capacidad del sujeto para utilizar recursos lingüísticos y expresar diferentes ideas, a través de la confrontación visual. 


\begin{tabular}{|c|c|c|c|c|c|c|}
\hline & $\begin{array}{l}\text { Core } \\
\text { Lan- } \\
\text { guage } \\
\text { CLS }\end{array}$ & $\begin{array}{l}\text { Recep- } \\
\text { tive } \\
\text { Lan- } \\
\text { guage } \\
\text { RLI }\end{array}$ & $\begin{array}{l}\text { Expre- } \\
\text { sive } \\
\text { Lan- } \\
\text { guage } \\
\text { ELI }\end{array}$ & $\begin{array}{l}\text { Language } \\
\text { Content } \\
\text { LCI }\end{array}$ & $\begin{array}{l}\text { Lan- } \\
\text { guage } \\
\text { Struc- } \\
\text { ture } \\
\text { LSI }\end{array}$ & $\begin{array}{c}\text { Working } \\
\text { Memory } \\
\text { WMI }\end{array}$ \\
\hline $\begin{array}{l}\text { Conceptos y siguiendo } \\
\text { direcciones }\end{array}$ & 4 & 4 & & 4 & & \\
\hline $\begin{array}{l}\text { Estructura de } \\
\text { palabras }\end{array}$ & 5 & & 5 & & 5 & \\
\hline Recordando oraciones & 10 & & 10 & & 10 & \\
\hline $\begin{array}{l}\text { Formulación de ora- } \\
\text { ciones }\end{array}$ & 5 & & 5 & & 5 & \\
\hline $\begin{array}{l}\text { Clases de palabras- } \\
\text { Receptivo }\end{array}$ & & 7 & & & & \\
\hline $\begin{array}{l}\text { Clases de palabras-To- } \\
\text { tal }\end{array}$ & & & & 18 & & \\
\hline $\begin{array}{l}\text { Estructura de oracio- } \\
\text { nes }\end{array}$ & & 7 & & & 7 & \\
\hline Vocabulario expresivo & & & & 15 & & \\
\hline $\begin{array}{l}\text { Repetición de núme- } \\
\text { ros-Total }\end{array}$ & & & & & & 7 \\
\hline Secuencias familiares & & & & & & 3 \\
\hline \multicolumn{7}{|l|}{$\begin{array}{l}\text { Recuento de puntua- } \\
\text { ciones }\end{array}$} \\
\hline $\begin{array}{l}\text { Suma de la puntua- } \\
\text { ción en los sub-test }\end{array}$ & 24 & 18 & 20 & 37 & 27 & 10 \\
\hline $\begin{array}{l}\text { Puntuación media } \\
\text { /Standard Score }\end{array}$ & 75 & 75 & 79 & 114 & 80 & 70 \\
\hline $\begin{array}{r}\text { Standard Score Points } \\
+/-\end{array}$ & 3 & 7 & 4 & 5 & 4 & 8 \\
\hline $\begin{array}{l}\text { Intervalo de con- } \\
\text { fianza(95\%Level) }\end{array}$ & 72 to 78 & 68 to 82 & 75 to 83 & $\begin{array}{c}109 \text { to } \\
119\end{array}$ & 76 to 84 & 62 to 78 \\
\hline Percentil & 5 & 5 & 8 & 82 & 9 & 2 \\
\hline $\begin{array}{l}\text { Distancia del prome- } \\
\text { dio }\end{array}$ & -1.5 & -1.5 & -1 & & -1 & -2 \\
\hline Clasificación & $\begin{array}{l}\text { Altera- } \\
\text { ción } \\
\text { mode- } \\
\text { rada }\end{array}$ & $\begin{array}{l}\text { Altera- } \\
\text { ción } \\
\text { mode- } \\
\text { rada }\end{array}$ & $\begin{array}{l}\text { Altera- } \\
\text { ción } \\
\text { leve }\end{array}$ & Sin altere & $\begin{array}{l}\text { Altera- } \\
\text { ción } \\
\text { leve }\end{array}$ & $\begin{array}{l}\text { Altera- } \\
\text { ción } \\
\text { severa }\end{array}$ \\
\hline
\end{tabular}

- Contenido: incluye aspectos semánticos relacionados con el vocabulario, desarrollo de conceptos y categorias, relación entre palabras, información inferencial, etc.

- Estructura del lenguaje: el sujeto señala la ilustración relacionada con la oración proporcionada.

- Memoria de trabajo: se mide el nivel de atención, concentración y capacidad de recuperar un término u oración clave.

Nuestro sujeto ha completado el nivel, obteniendo las siguientes puntuaciones: 
- Lenguaje básico (Core Language): 24/75

- $\quad$ Lenguaje receptivo: $18 / 75$

- $\quad$ Lenguaje expresivo: 20/75

- Contenido: $37 / 114$

- Estructura del lenguaje: 27/80

- Memoria de trabajo: 10/70

Como podemos observar, las pruebas donde el sujeto ha obtenido una puntuación más baja han sido en Contenido, Memoria de trabajo y lenguaje receptivo.

- En el Nivel 3 de esta prueba, los sub-test van a ir encaminados al análisis de las "conductas clinicas subyacentes"; es decir, a través de los sub-test que incorpora, se van a evaluar los aspectos y comportamientos clinicos más frecuentes que derivan de los trastornos del lenguaje observados en el paciente. Las diferentes subpruebas proporcionadas para este nivel son:

- Conciencia fonológica: mide la capacidad del sujeto para conocer y manipular los fonemas del lenguaje ya sea a nivel silábico, de la palabra u oración.

En este sub-test el sujeto obtiene una puntuación de 39. Según las normas de puntuación, los valores inferiores a 47 indican un desempeño inferior a lo esperado para la edad.

- Asociación de palabras: el sujeto tiene que nombrar conceptos dentro de una determinada categoría mientras es cronometrado.

En este caso, la puntuación conseguida es 19; los valores inferiores a 25 indican un desempeño inferior a lo esperado para la edad.

- Denominación automática rápida: el sujeto tiene que nombrar colores, acciones, formas, mientras está siendo cronometrado.

En esta subprueba el sujeto obtiene un desempeño atípico, puesto que necesita más de 100 segundos para terminar la tarea proporcionada. Respecto a los errores, también hemos observado que el número de errores supera lo esperado para su edad.

- Memoria de trabajo: el sujeto cuenta hacia delante y luego hacia atrás, dice los días de la semana, meses del año, etc.

Es uno de los procesos en los que el sujeto ha obtenido resultados más bajos su calificación es de alteración severa. 
- Finalmente, el Nivel 4 evalúa el lenguaje y la comunicación del sujeto dentro de un contexto, a través de dos sub-test:

- Perfil pragmático: con este sub-test obtenemos información sobre la capacidad de uso lingüístico del sujeto a través de una lista de elementos descriptivos, que se enmarcan en tres áreas: destrezas conversacionales y rituales; pedir, dar o responder a cierta información, y habilidades en comunicación no verbal.

- Escala de Valoración del Lenguaje: muestra una visión sobre ciertas habilidades comunicativas: escucha, habla y la lectura.

En lo que compete a este último sub-test, nuestro sujeto no presenta problemas de audición ni comprensión de mensajes orales y expresiones del lenguaje no verbal. Sí es frecuente, sin embargo, que pida que le repitan aquello que se le ha dicho. Por otro lado, muestra un gran déficit de atención, por ello en ocasiones le resulta imposible seguir el hilo comunicativo en una conversación.

Su vocabulario no es variado y en numerosas ocasiones no encuentra la palabra correcta que quiere decir (anomia frecuente). Esta imposibilidad de recuperar palabras y conceptos influye a la hora de describir elementos, expresar sus pensamientos, así como poner orden a los hechos que narra. Todo ello va a influir en su capacidad comunicativa, pero, además, en el aprendizaje en general y en la lecto-escritura, en particular (Bruner, 1981).

\section{REgISTRO FONOLÓGICO INDUCIDO}

La segunda prueba propuesta para el estudio fonológico de nuestro sujeto es el test Registro Fonológico Inducido (Monfort y Juárez, 2010). Dicho test está compuesto por 57 ítems o términos clave (Cuadro 1); cada ítem está representado a través de una imagen reconocible para el sujeto. Los términos propuestos en la prueba cubren el espectro fonológico fundamental del español. El objetivo de esta prueba, por tanto, es analizar las enunciaciones que el sujeto realiza a través de la confrontación visual.

\begin{tabular}{|l|l|l|l|l|}
\hline 1. moto & 13.indio & 25.sol & 37.palmera & 49.gorro \\
\hline 2. boca & 14.toalla & 26.casa & 38.clavo & 50.rata \\
\hline 3. piña & 15.fuma & 27.pez & 39.tortuga & 51.cabra \\
\hline 4. piano & 16.dedo & 28.jaula & 40.pueblo & 52.lavadora \\
\hline 5. pala & 17.peine & 29.zapato & 41.tambor & 53.preso \\
\hline 6. pie & 18.ducha & 30.flan & 42.escoba & 54.semáforo \\
\hline 7. niño & 19.gafas & 31.lápiz & 43.mariposa & 55.fresa \\
\hline 8. pan & 20.toro & 32.pistola & 44.puerta & 56.árbol \\
\hline 9. ojo & 21.silla & 33.mar & 45.bruja & 57.periódico \\
\hline 10.1lave & 22.taza & 34.caramelo & 46.grifo & \multirow{2}{|}{} \\
\hline 11.luna & 23.cuchara & 35.plátano & 47.jarra & \\
\cline { 1 - 3 } 12.campana & 24.teléfono & 36.globo & 48.tren &
\end{tabular}

Tabla 1: Registro Fonológico Inducido. 
Una vez realizada la prueba, se han obtenido 17 producciones alteradas de los 57 elementos. Entre estas alteraciones, sería interesante destacar ciertos comportamientos lingüisticos que mencionamos a continuación:

- Frecuente simplificación de diptongos (CVV pasa a ser CV), como en pue$b l o \rightarrow$ [peblo], puerta $\rightarrow$ [perta], periódico $\rightarrow$ [perodico].

- Asimilación lateral como en caramelo $\rightarrow$ [kalamelo], árbol $\rightarrow$ [álbol].

- Duplicación de sílabas como en mariposa $\rightarrow$ [maripoposa], teléfono $\rightarrow$ [telelefono].

- Simplificación del ataque complejo: pistola $\rightarrow$ [pitola].

Sin embargo, cabe resaltar que el sujeto no muestra ninguna dificultad en la realización de los sonidos vibrantes [rr] y [r]; siendo capaz de producir correctamente términos como gorro, jarra, tortuga, mariposa, periódico.

\section{EVALUACIÓN FONOLÓGICA DEL HABLA INFANTIL. LAURA BOSCH}

Esta prueba está compuesta por un total de 62 elementos fonéticos, distribuidos en 32 términos que el individuo tiene que producir oralmente por confrontación visual. Gracias a este test podemos analizar el desarrollo fonológico del sujeto y diagnosticar posibles problemas articulatorios.

La prueba está dividida en cuatro secciones diferentes; en primer lugar, tenemos la evaluación fonológica, donde se agrupan los diferentes términos para ir anotando las producciones del individuo. A continuación, se estudia el perfil fonológico, donde vienen recogidos los fonemas consonánticos del español, codas, grupos consonánticos y diptongos presentes en la prueba; además, en esta sección contamos con una enumeración de procesos sistémicos (lateralización de vibrantes, insonorización, oclusivización de fricativas, etc.) y estructurales (omisión de codas, simplificación de diptongos, reduplicaciones silábicas, etc.) (Bosch Galcerán, 1983:10-11) que el sujeto puede producir al realizar el test. El tercer apartado está dedicado al repertorio fonético. Aquí vamos a recoger todos aquellos sonidos que nuestro individuo es capaz de emitir y, por tanto, están presentes en su repertorio fonético. En este apartado se incluye además un análisis detallado de las estructuras silábicas presentes en la prueba, de manera que podemos anotar el número de alteraciones estructurales que realiza el sujeto. Por último, en el apartado de procesos fonológicos de simplificación, se van a analizar los diferentes procesos de asimilaciones presentes en las producciones obtenidas.

Tras la realización del test, hemos observado los siguientes resultados:

A) De los 32 ítems presentados, el sujeto ha realizado un total de 12 producciones alteradas. Estas alteraciones han sido agrupadas atendiendo al proceso de simplificación o asimilación que han sufrido: 
- Simplificación de diptongos: fuego $\rightarrow$ [fego]; piedra $\rightarrow$ [pedra]

- $(\mathrm{CVV} \rightarrow \mathrm{CV})$.

- Simplificación del ataque complejo: clase $\rightarrow$ [kase] (CCV $\rightarrow \mathrm{CV})$; cristal $\rightarrow$ [kistal] $(\mathrm{CCVC} \rightarrow \mathrm{CVC})$.

- Estridencia: taza $\rightarrow$ [tasa].

B) Con esta prueba hemos descartado la presencia de algún trastorno articulatorio y además hemos podido reafirmar que el sujeto es capaz de producir los sonidos [r y rr], lo cual nos parece interesante ya que, teniendo en cuenta la edad del sujeto, cabría esperar que estos fueran los sonidos donde presentara más dificultades (Susaníbar Chávez et al., 2013: 26-27).

C) De forma paralela, hemos ido observando una frecuente simplificación que nuestro sujeto realiza en los diptongos propuestos en las diferentes pruebas realizadas, pero también en el habla espontánea. Este hecho ha motivado la creación de una prueba, cuyo objetivo es, precisamente, el análisis de las producciones en términos que incluyan diptongos y triptongos.

\section{PRUEBA FONOLÓGICA DE DIPTONGOS Y TRIPTONGOS}

Los resultados e ideas anteriores han dado como resultado la creación de la Prueba fonológica de diptongos y triptongos. Para su elaboración, hemos seleccionado los diptongos y triptongos presentes en el español, en posición inicial, media y final de palabra, y en silabas átonas y tónicas. Estos diptongos y triptongos han sido insertados en pseudo-palabras, esto es, palabras inventadas, cuyo fin es eliminar cualquier estimulo que facilite el reconocimiento del término y, por ende, su pronunciación.

\subsection{PRUEBa FONOLÓGICA DIPTONGOS}

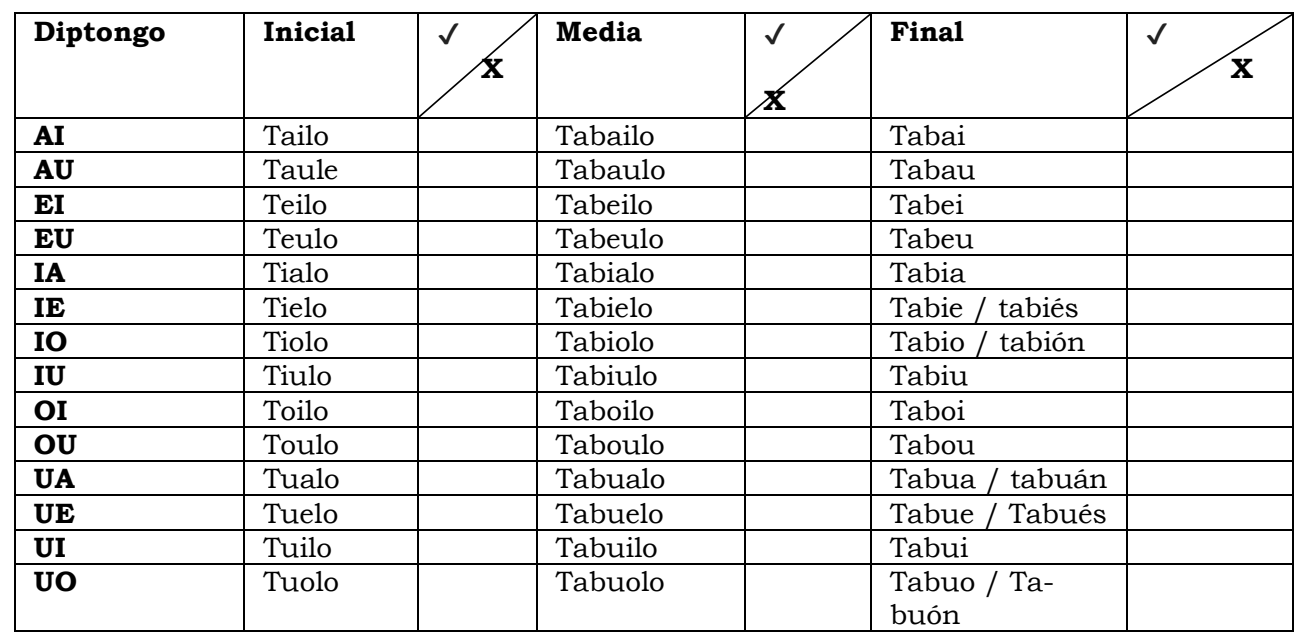




\subsection{PRUEBA FONOLÓgICA TRIPTONGOS}

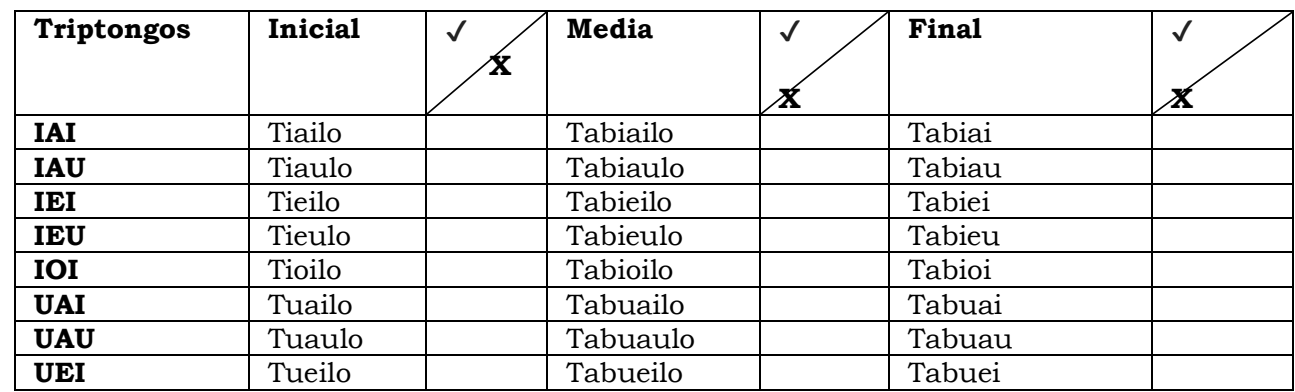

Debido a las dificultades que nuestro sujeto presenta en la lectoescritura, ha sido necesario facilitarle los diferentes términos, para que este los repita. Aunque este hecho resta espontaneidad, ya que el sujeto recibe el ítem para producir, es posible observar y analizar las dificultades, o la ausencia de ellas, en lo que compete a la alteración de la estructura silábica en términos con diptongos y triptongos.

Tras la realización de la prueba, hemos observado que nuestro sujeto no presenta problemas al repetir los términos, obteniendo una única producción alterada en cada prueba:

- Prueba de diptongos: Taboilo (diptongo decreciente, posición media) $\rightarrow$ [tabilo].

$$
\mathrm{CVV} \rightarrow \mathrm{CV}
$$

- Prueba triptongos: Tabiaulo (posición media) $\rightarrow$ [Tabiulo].

$$
\mathrm{CVVV} \rightarrow \mathrm{CVV}
$$

Sería interesante analizar las estructuras silábicas de términos con diptongos y triptongos en ítems que el sujeto pueda leer sin ayuda de un tercero. Esta prueba se realizará en un futuro estudio.

\section{CONCLUSIONES Y FUTURAS LÍNEAS DE INVESTIGACIÓN.}

Este trabajo se enmarca dentro de una investigación a un paciente con microduplicación en el cromosoma 1.21q1. Esta investigación tiene como objetivo último la elaboración de un perfil lingüístico-comunicativo del paciente, que sirva tanto a especialistas, como a la propia familia en la comprensión de los trastornos a nivel de habla derivadas de la alteración genética observada.

Las diferentes pruebas presentadas han servido para descartar cualquier posible disfunción articulatoria en nuestro sujeto. Por otro lado, hemos podido observar, basándonos en los datos obtenidos, que esta persona ha adquirido los fonemas de acuerdo con su etapa y que la capacidad de repetir 
sonidos, alterados en conversación espontánea. Sin embargo, sí se puede afirmar que existan alteraciones fonológicas en sus producciones como así hemos observado en la simplificación de grupos consonánticos, asimilaciones y reducción de diptongos.

Finalmente, teniendo en cuenta que el gen ROBO1 se encuentra sobreexpresado, y que nuestro sujeto se encuentra en la etapa donde se suele desarrollar la lecto-escritura (Espacio logopédico 1, 2015), sería interesante estudiar cómo el niño adquiere dicha capacidad. De igual forma, será importante observar cómo evolucionan las alteraciones fonético-fonológicas descritas en este estudio y, además, analizar el componente cognitivo y su evolución, ya que lenguaje y cognición son capacidades humanas estrechamente relacionadas que contribuyen a la capacidad comunicativa del individuo (Bermúdez y a González, 2011).

\section{REFERENCIAS}

BENÍTEZ BURRACO, A. (2006): "La evolución de los genes del lenguaje", Interlingüística, 16, pp. 189-204.

BENÍTEZ BURRACO, A. (2007): “Aspectos genéticos del lenguaje”, Revista Española de Lingüistica, 37 (1), pp. 103-117.

BENÍTEZ BURRACO, A. et al. (2018): "Narrowing the genetic causes of language dysfunction in the 1q21.1 microduplication sindrome", Frontiers in Pediatrics, 6, p. 163.

BERMÚDEZ, L. y GONZÁLEZ, L. (2011): "La competencia comunicativa: elemento clave en las organizaciones", Quórum Académico, 8, 15, pp. 95-110.

BOSCH GALCERAN, L. (1983): "El desarrollo fonológico infantil: una prueba para su evaluación", Anuario de Psicologia, 28 (1), pp. 10-11.

BOSCH GALCERAN, L. (2004): Evaluación fonológica del habla infantil, Barcelona: Masson.

BRUNER, J. (1981): "De la comunicación al lenguaje. Infancia $\mathrm{y}$ Aprendizaje", Journal for the Study of Education and Development, 4, pp. 33-63.
ESPACIO LOGOPÉDICO (2015): Lectoescritura a qué edad. Disponible en: https://www.espaciologopedico.com/noticias/det/6495/lectoescrituraa-que-edad.html (Fecha de consulta: 20/01/2019).

GENETIC AND RARE DISEASE DEPARTAMENT (G.A.R.D) (2013): Chromosome 1q21.1 duplication syndrome. Disponible en: https://rarediseases.info.nih.gov/diseases/10591/chromosome1q211-duplication-syndrome (Fecha de consulta: 25/11/2018).

MONFORT, M. y JUÁREZ, A. (2010): Registro Fonológico Inducido, Madrid: Editorial CEPE.

PULVERMÜLLER, F. (2002). The Neuroscience of Language. On Brain Circuits of Words and Serial Order, Cambridge: University Press. Cambridge, pp. 18-24.

SUSANÍBAR CHÁVEZ, F. et al. (2013): "Adquisición fonética-fonológica", Revista Digital EOS Perú, 1 (1), pp. 26-27.

UNIQUE RARE CHROMOSOME DISORDER SUPPORT (2010): Microduplicaciones 1q21.1. 
Disponible en: https:/ /www.rarechromo.org/media/translations/Espanol/1q21.1\%20Microduplicaciones $\% 20$ Spanish\%20FTNW.pdf (Fecha de consulta: $12 / 11 / 2018)$.
WIIG, E. H. et al. (2006): Clinical Evaluation of Language Fundamentals - Fourth Edition, Spanish (CELF-4 Spanish), U.S.A.: Pearson. 